\title{
Sinonimia y variación léxica en la fraseología española e italiana: propuesta para un diccionario bilingüe bidireccional en línea
}

\author{
Elena Dal Maso ${ }^{1}$ \\ Recibido: 30 de noviembre de 2019 / Aceptado: 7 de abril de 2020.
}

\begin{abstract}
Resumen. En el presente artículo se ilustra una propuesta enfocada al tratamiento de la variación léxica y de la sinonimia en un diccionario fraseológico en línea en curso de elaboración. El objetivo de dicha propuesta es doble: por un lado, plantear una posible estrategia para dar cuenta de la variación léxica y la sinonimia en el ámbito fraseográfico, $\mathrm{y}$, por otro, contribuir a solucionar algunas de las lagunas existentes hasta la fecha en la fraseografía bilingüe del italiano y del español mediante muestras concretas de fichas pensadas para el medio digital. Como resultado, en los apartados que configuran este ensayo se aclara, en primer lugar, la metodología adoptada para la selección y el análisis del corpus estudiado; en segundo lugar, se ofrecen las fichas lexicográficas, todas ellas elaboradas a partir del concepto de equivalencia funcional (Mellado Blanco 2015) y de la propuesta lexicográfica expuesta en Lončar y Dal Maso (2018) y Dal Maso (2019).
\end{abstract}

Palabras clave: fraseología bilingüe; herramientas digitales; variación léxica; sinonimia

[en] Synonymy and lexical variation in Spanish and Italian phraseology: proposal for an online bilingual bidirectional dictionary

\begin{abstract}
This article presents a lexicographical proposal focused on lexical variation and synonymy in Spanish and Italian idioms. The purpose of this proposal is twofold: firstly, to put forward a possible strategy for the lemmatization of lexical variation and synonymy in dictionaries of idioms, and, secondly, to contribute to the development of Spanish/Italian bilingual phraseography by offering some patterns for lexicographical records in digital format. As a result, in the first part of this essay we describe the methodology used in order to select and analyse the idioms; then, in the second part, we propose lexicographical records for the elaboration of an online dictionary of idioms - currently in progress -based on the functional equivalence criterion (Mellado Blanco 2015) and the lexicographical proposal illustrated in Lončar and Dal Maso (2018) y Dal Maso (2019).
\end{abstract}

Keywords: bilingual phraseology; digital tools; lexical variation; synonymy

Índice. 1. Introducción. 2. Corpus y metodología. 3. Variación léxica y sinonimia: propuesta para un diccionario fraseológico bilingüe bidireccional en línea. 3.1. Variantes léxicas excluyentes y facultativas. 3.2. Sinonimia. 4. Conclusiones. Agradecimientos. Bibliografía

Cómo citar: Dal Maso, E. (2020). Sinonimia y variación léxica en la fraseología española e italiana: propuesta para un diccionario bilingüe bidireccional en línea. En Representación de la fraseología en herramientas digitales: problemas, avances, propuestas, M. De Beni (ed.), Círculo de Lingüística Aplicada a la Comunicación 82, 27-40, https://dx.doi.org/10.5209/clac.689561

\section{Introducción}

La aplicación de las nuevas tecnologías a la lexicografía ha supuesto en las últimas décadas transformaciones profundas en la confección y la consulta de diccionarios; entre otras, destaca la oportunidad de actualizar constantemente la información contenida tanto en la macro- como en la microestructura y de incluir en las entradas lexicográficas vínculos hipertextuales y una mayor cantidad de datos. También merece la pena señalar otra ventaja que consiste en ofrecer "datos dinámicos en función de las necesidades" de los usuarios a los que un diccionario va dirigido (Nomdedeu-Rull y Tarp 2018: 2). Por otra parte, la estabilidad y

\footnotetext{
${ }^{1}$ Università Ca’ Foscari Venezia. Correo electrónico: elena.dalmaso@unive.it
} 
durabilidad de estos nuevos formatos, junto con la fiabilidad de los contenidos propuestos, constituyen puntos débiles sobre los que sería conveniente reflexionar con atención (sobre los logros y los peligros de los diccionarios digitales véanse, entre otros, Gelpí Arroyo 2003, Águila Escobar 2009 y Marello 2017).

De las ventajas que ofrece el soporte electrónico se está sirviendo con provecho no solamente la lexicografía general — sobre todo monolingüe-, sino también la fraseografía, tal y como demuestra el Diccionario de Locuciones del Español Actual (DiLEA), puesto a disposición recientemente por Penadés Martínez (2019). Efectivamente, la falta de vínculos espaciales permite solucionar muchos retos pendientes en lo que a la lematización de las unidades fraseológicas se refiere; piénsese, por ejemplo, en el tratamiento de la polisemia — analizado en Lončar y Dal Maso (2018) y Dal Maso (2019), entre otros- o bien de las variantes y los sinónimos fraseológicos. Como es bien sabido, sobre estos dos últimos fenómenos se han derramado ríos de tinta (señalamos, entre otros, Zuluaga Ospina 1980, Corpas Pastor 1996, Álvarez de la Granja 1999, Montoro del Arco 2004, Penadés Martínez 2006, García-Page Sánchez 2008 y 2016, Rodríguez-Piñero Alcalá 2012, Fajardo Rojas 2017) y aún persisten hoy en día muchas dudas en cuanto a su definición. A esto cabe añadir la existencia de casos que se hallan a medio camino entre la variación y la sinonimia, de ahí que sea legítimo preguntarse si se trata de dos nociones distintas o más bien de un continuum que abarca las posibles manifestaciones, semánticas o bien formales, de la identidad y la alteridad en las UF (García-Page Sánchez 2008: 221).

Por lo que concierne al panorama fraseográfico actual, aparte de los diccionarios fraseológicos existentes, son muy escasos los estudios que han abordado con propuestas prácticas la representación lexicográfica de estos dos conceptos, sobre todo en relación con el español y el italiano (véanse, por ejemplo, RodríguezPiñero Alcalá 2011 para el español, Imperiale y Schafroth 2019 y Autelli, Konecny y Lusito 2019 para el italiano). La escasez de planteamientos fraseográficos resulta aún más evidente en el marco contrastivo entre estos dos idiomas. De hecho, frente a la abundancia de diccionarios bilingües generales (considérese la lista que se ofrece en el Portal de lingüística contrastiva español-italiano, dirigido por San Vicente) y de aportaciones teóricas a la fraseografía ítalo-española (Quiroga Munguía 2005, Navarro 2008, Valero Gisbert 2010, 2012, 2015, 2017, 2019, Capra 2016a, 2016b, entre otros), destaca la publicación de tan solo dos diccionarios fraseológicos bilingües -Spagnolo idiomatico. Dizionario spagnolo italiano di frasi idiomatiche, colloquiali e gergali (2013) de Sañé y Schepisi y el Diccionario combinatorio español italiano (A-K) (2019) de Simone Greco, en el que se registran, junto a otras combinaciones frecuentes, numerosas colocaciones y locuciones-, ambos en papel y monodireccionales (del español al italiano).

Así las cosas, en el presente trabajo se ilustra una propuesta dedicada al tratamiento lexicográfico de la variación y de la sinonimia fraseológicas entre español e italiano, surgida en el marco del proyecto de investigación Diccionario fraseológico multilingüe digital (DFMD), que se está llevando a cabo en la Universidad Ca' Foscari de Venecia en colaboración con Ivana Lončar, de la Universidad de Zadar, y Pilar Valero Fernández, de la Universidad de Castilla-La Mancha. El diccionario planteado en este proyecto contempla, en su fase inicial, la lematización de las unidades fraseológicas del español peninsular, del croata y del italiano; aun así, no se excluye la posibilidad de ampliar el abanico de idiomas considerados en otras etapas sucesivas. Puesto que quien suscribe el presente artículo se dedica a la extracción de la macroestructura y a la elaboración de la planta lexicográfica relativas al español y al italiano, la metodología que se describe y aplica en las páginas siguientes está enfocada en el desarrollo de un diccionario bilingüe bidireccional —esto es, una herramienta provechosa para "due comunità linguistiche parlanti lingue materne diverse" y caracterizada por presentar "una sezione lingua $\mathrm{x} \rightarrow$ lingua y e un'altra sezione lingua y $\rightarrow$ lingua x" (Marello 2017: 203) — que, a su vez, formará parte del DFMD.

A la luz de ello, el objetivo del estudio que se presenta es doble: por un lado, plantear una posible estrategia para dar cuenta de la variación léxica y de la sinonimia en los diccionarios fraseológicos en línea; por otro, contribuir a solucionar algunas de las lagunas existentes hasta la fecha en la fraseografía bilingüe bidireccional del italiano y del español mediante muestras concretas de fichas. Como resultado, en los apartados que configuran este ensayo se aclara, en primer lugar, la metodología adoptada para la recopilación y el análisis del corpus estudiado; en segundo lugar, se ofrece una muestra de fichas lexicográficas, todas ellas elaboradas a partir del concepto de equivalencia funcional (Mellado Blanco 2015) y de la propuesta lexicográfica expuesta en Lončar y Dal Maso (2018) y en Dal Maso (2019).

\section{Corpus y metodología}

Para la selección del corpus fraseológico que se considera en este trabajo, nos hemos basado principalmente en las nociones de variante léxica y sinonimia enunciadas por García-Page Sánchez (2008 y 2016) y Fajardo Rojas (2017). En concreto, coincidimos con el primer estudioso (García-Page Sánchez 2008: 219) en definir 
como variantes fraseológicas aquellas "modulaciones formales [...] codificadas o institucionalizadas" que puede manifestar un fraseologismo bien en los planos fónico (a volapié / a vuelapié) y ortográfico ( $a$ matacaballo / a mata caballo), bien en los niveles de análisis morfológico (ojo/ojos de lince), gramatical (estar con el agua al/hasta el cuello), sintáctico (hacer oídos sordos/de mercader) o léxico (rata/ratón de biblioteca). En este último caso, la variación consiste en la posibilidad de conmutar lexemas que "son siempre sinónimos desde el punto de vista fraseológico, pero no siempre desde el punto de vista semántico" (García-Page Sánchez 2008: 244); compárense, a este propósito, las locuciones esp. enseñar/mostrar los dientes y con el corazón en la boca/garganta, que incluyen respectivamente dos verbos sinónimos (enseñar y mostrar) y dos sustantivos que no son sinónimos (boca y garganta). En consecuencia, quedan excluidas de nuestra propuesta las conmutaciones que sí afectan al significado de una UF y que algunos diccionarios (p. ej. Buitrago 2009, 3. ${ }^{a}$ ed.) incluyen en el conjunto de las variantes léxicas: este es el caso de esp. apostar/jugar a caballo ganador y apostar/jugar a caballo perdedor, así como de sus equivalentes italianos puntare/scommettere sul cavallo vincente e puntare/scommettere sul cavallo perdente, que en nuestro repertorio configuran cuatro entradas distintas, dos para el español y dos para el italiano. No se trata, pues, de variantes léxicas de una misma UF, sino de pares de antónimos fraseológicos.

Además de las variantes por conmutación, existen otras que son el producto de una operación de adición o sustracción (García-Page Sánchez 2008: 219); de ahí la diferenciación, delineada por Larreta Zulategui (2001: 92), entre variantes excluyentes —-denominadas así porque "la realización de una de ellas implica automáticamente la exclusión de la otra", como en esp. hueso duro/difícil de roer- y variantes facultativas - cuya presencia es opcional y no altera el significado de la unidad, como en it. (bella/brutta) gatta da pelare-. García-Page Sánchez (2008: 238), por su parte, denomina este segundo tipo de variante abreviatura fraseológica y lo ubica en el nivel de la variación sintáctica. Aun así, en este trabajo, tanto las variantes excluyentes como las facultativas se engloban en la noción de variación léxica, siendo de hecho añadiduras que repercuten numéricamente en los constituyentes fijos de un fraseologismo sin llegar a alterar la función sintáctica que estos desempeñan dentro de la misma unidad fraseológica. De ahí que en nuestras fichas se ejemplifiquen ambas tipologías.

A diferencia de la variación, la sinonimia — considerada en su vertiente interfraseológica, según la denomina García-Page Sánchez (2008: 220) - supone que dos o más UF sean a un tiempo "formalmente distintas y semánticamente equivalentes". Sin embargo, el grado de identidad semántica debe concebirse en términos no absolutos, ya que en ocasiones las unidades sinónimas pueden poseer un significado muy parecido aun mostrando "alguna restricción de orden estilístico, sociolectal o geolectal, alguna diferencia en el grado de intensidad, la ausencia [...] de un rasgo sémico secundario presente en la sinonimia, etc." (García-Page Sánchez 2008: 398). En los diccionarios considerados en su trabajo — concretamente, el Diccionario ideológico de la lengua española (1942) de Casares, la vigesimosegunda edición del diccionario académico (2001), el Diccionario del español actual (1999) y el Diccionario fraseológico documentado del español actual (2004), estos últimos publicados por Seco, Andrés y Ramos-, el mismo estudioso (2008: 399-401) observa que a fraseologismos sinónimos se asocian a menudo definiciones no del todo coincidentes.

Otra propuesta que se ha tenido en cuenta es la de Fajardo Rojas (2017: 95), según la cual los parámetros que permiten identificar los sinónimos fraseológicos son: 1. la equivalencia semántica; 2 . la pertenencia a una misma categoría gramatical; 3. la discrepancia formal; 4. la posibilidad de intercambio en un mismo contexto. Como resultado, pueden considerarse sinónimas las locuciones esp. salir por patas, poner pies en polvorosa y tomar las de Villadiego, que significan 'marcharse muy deprisa'; y también encajarían en este fenómeno los equivalentes it. darsela a gambe, scappare a gambe levate y tagliare la corda. Dichos parámetros facilitan en la práctica la distinción entre sinonimia y variación, esta última identificable, según la misma autora (Fajardo Rojas 2017: 96), por la falta de cambios sintagmáticos y semánticos en la UF examinada.

Con el fin de recopilar el corpus de locuciones que se analiza en los apartados 3.1 y 3.2 , se han consultado, en primer lugar, diccionarios monolingües españoles (DLE 2014; DUE 2007, 3. ${ }^{\text {a }}$ ed.) e italianos (GRADIT 1999-2007; Vocabolario Treccani 2008), diccionarios bilingües bidireccionales españolitaliano/italiano-español (Arqués y Padoan 2012; Tam 2004, 3. a ed.), bilingües monodireccionales españolitaliano (Sañé y Schepisi 2013; Greco 2019) y, finalmente, diccionarios fraseológicos españoles (Buitrago 2009, 3. ${ }^{\text {a }}$ ed.; Seco, Andrés y Ramos 2004; Varela y Kubarth 2004, 2. ${ }^{\mathrm{a}}$ ed.; DiLEA 2019) e italianos (Pittano 2009; Quartu y Rossi 2012, 2. ${ }^{\text {a }}$ ed.; Sorge 2011, 3. a ed.). Sucesivamente, hemos averiguado el significado y el uso de las UF seleccionadas, así como su actualización formal en el discurso, mediante la consulta de las ediciones electrónicas de periódicos españoles e italianos, tanto nacionales como locales (El País, El Mundo, 
La Vanguardia, Diario de Burgos, La Repubblica, Il Fatto Quotidinano Il Sole 24 ORE, Corriere Adriatico, entre otros), de los corpus textuales CORPES XXI para el español y PAISÀ (2009-2012, coordinado por Scalise y Pirrelli) para el italiano - que registran, en ambos casos, fragmentos procedentes tanto de textos escritos como de fuentes multimodales u orales (sobre todo de canales televisivos o radiofónicos) - y de otras páginas web corporativas e informativas, blogs y foros.

Una vez recopilado el corpus, el paso siguiente ha sido la aplicación del criterio de equivalencia funcional, cuyo fin es establecer correspondencias interlingüísticas prototípicas y provechosas tanto en el ámbito lexicográfico como en la glotodidáctica y en la mayoría de las situaciones comunicativas. Según asevera Mellado Blanco (2015: 155), la equivalencia funcional, por un lado, "se diferenciaría de la equivalencia sistémica en que no se limita a describir pares de unidades fraseológicas descontextualizadas, sino que considera las situaciones de uso y contempla además la posibilidad de equivalencia mediante un lexema simple"; por otro, "se diferencia de la equivalencia textual en que no pretende hacerse eco de toda la casuística posible de traducciones de un fraseologismo de la L1 en la L2" (2015: 155). En consecuencia, los tres parámetros que más inciden en la elaboración de las correspondencias funcionales son la equivalencia de los fraseologismos en el plano semántico, el componente connotativo y pragmático y, para acabar, la valencia sintáctica y semántica (Mellado Blanco 2015: 164-169). La suma de estas tres variables da como resultado la elaboración de equivalencias adecuadas no solamente desde el punto de vista del significado, sino también por lo que concierne a cuestiones como la variación diafásica o diastrática, la frecuencia de uso y la combinatoria con actantes externos (p. ej., el sujeto o el complemento directo de una locución verbal). Por el contrario, otros criterios como los componentes léxicos, la imagen subyacente, la estructura morfosintáctica y la función sintáctica, que sí serían pertinentes a nivel de sistema, dejan de ser relevantes si el objetivo del estudio contrastivo es de tipo funcional o textual (Mellado Blanco 2015: 160). Debido a ello, en la propuesta que presentamos a continuación se han tenido en cuenta ante todo los tres parámetros semánticos y pragmáticos mencionados anteriormente, que se han aplicado tanto a los conjuntos de locuciones con variantes léxicas como a los que presentan sinonimia.

\section{Variación léxica y sinonimia: propuesta para un diccionario fraseológico bilingüe bidireccional en línea}

Las fichas que se proponen en este apartado ejemplifican la planta lexicográfica delineada por primera vez en Lončar y Dal Maso (2018) y sucesivamente aplicada al tratamiento de la polisemia simétrica y asimétrica en Dal Maso (2019).

De entrada, cabe destacar que las locuciones lematizadas en nuestra propuesta se hallan bajo todas las palabras léxicas que las configuran, de ahí que, por ejemplo, la UF esp. apostar/jugar a caballo ganador aparezca tanto bajo los dos verbos apostar y jugar como bajo caballo y ganador. En una herramienta digital como la que estamos construyendo, la falta de límites de espacio físico y la oportunidad de registrar bajo más lemas una misma locución facilitan, según se defiende, el proceso de consulta de los contenidos recopilados, puesto que ofrece al usuario múltiples caminos para llegar a la entrada objeto de su búsqueda, evitando, por otra parte, el uso de remisiones que dilatarían el tiempo necesario para finalizar la averiguación lexicográfica. Por esta razón también se ha decidido agrupar bajo el mismo núcleo nominal todos aquellos fraseologismos que lo contienen, prescindiendo del género o del número con el que este se actualice en cada caso; por tanto, al buscar it. gatto, - $a$ el usuario encontrará tanto (essere come) cane e gatto como (bella/brutta) gatta da pelare o quattro gatti, entre otros. La lematización conjunta de las UF que comparten el mismo sustantivo también favorece el contraste interlingüístico e intercultural, al dar cuenta del papel —a menudo simbólico- atribuido por una sociedad determinada a los elementos de su entorno o de su sistema de creencias y conocimientos.

Por lo que concierne a la forma canónica de las UF, en la planta lexicográfica que ilustramos en este apartado se ha optado por señalar en negrita los constituyentes fijos y en redonda los actantes obligatorios externos. Tras la forma canónica, en cada ficha lexicográfica aparecen, en este orden, los siguientes elementos: la categoría gramatical, posibles indicaciones sobre la combinatoria o las restricciones de uso (entre barras oblicuas), el significado y, finalmente, al menos dos muestras de uso procedentes de la prensa digital y los corpus textuales consultados. Si una locución se actualiza en el discurso con dos o más variantes léxicas, se proponen testimonios auténticos para cada variante. De forma contraria, si el fraseologismo en cuestión no presenta variantes, en la ficha se registran dos ejemplos de uso.

Las muestras seleccionadas para este trabajo son todas relativas al intervalo temporal incluido entre enero de 2015 y octubre de 2019 y tienen el propósito de fomentar la comprensión y el uso activo de las locuciones ofreciendo ejemplos prototípicos en cuanto al uso y al significado de los fraseologismos e inteligibles a pesar 
del tema específico abordado en el texto de procedencia. Pulsando en las referencias de cada muestra, el usuario podrá acceder al texto original mediante un salto reticular.

La misma metodología se adopta en la segunda parte de la ficha, en la que se proporciona la información lexicográfica relativa al equivalente (o los equivalentes) en la lengua de llegada. En la propuesta que se detalla a continuación, en ocasiones aparece un apartado final de observaciones que recoge, entre otros detalles, uno o más antónimos fraseológicos en la lengua meta, siempre y cuando el significado de la locución y de su equivalente funcional contemple la existencia de un significado opuesto. Las fichas completas de las unidades ubicadas en dicho apartado podrán visualizarse seleccionando con el cursor las respectivas formas canónicas.

En la sección final también se deja constancia de los fraseologismos sinónimos en la lengua de partida, siempre que existan; en cambio, las unidades sinónimas en el idioma de llegada se incluirán, como veremos más adelante, en el cuerpo de la ficha para favorecer la elección del equivalente funcional más adecuado. Esta diferenciación en el tratamiento de la sinonimia, así como la inclusión de antónimos en el apartado final de observaciones, corresponde a la voluntad de beneficiar a los usuarios potenciales — sobre todo estudiantes y traductores - de una herramienta como la que ilustramos aquí, proporcionando datos claros y lo más completos posible sobre un fraseologismo determinado y sus relaciones intra- e interlingüísticas con otras unidades de las lenguas consideradas.

\subsection{Variantes léxicas excluyentes y facultativas}

Nuestro proyecto fraseográfico prevé que las variantes léxicas excluyentes y facultativas se incorporen a las formas canónicas de los fraseologismos mediante recursos gráficos distintos. En concreto, las variantes excluyentes aparecen separadas por una barra oblicua si se trata de elementos que no coinciden con la palabra léxica que el usuario teclea en el buscador del diccionario — es decir, el núcleo de la macroentrada. Considérese, por ejemplo, el caso de esp. apostar/jugar a caballo ganador tal y como aparecería bajo caballo y ganador (de hecho, la única diferencia entre las dos fichas correspondería a la presencia de un núcleo distinto en la macroentrada, respectivamente caballo y ganador):

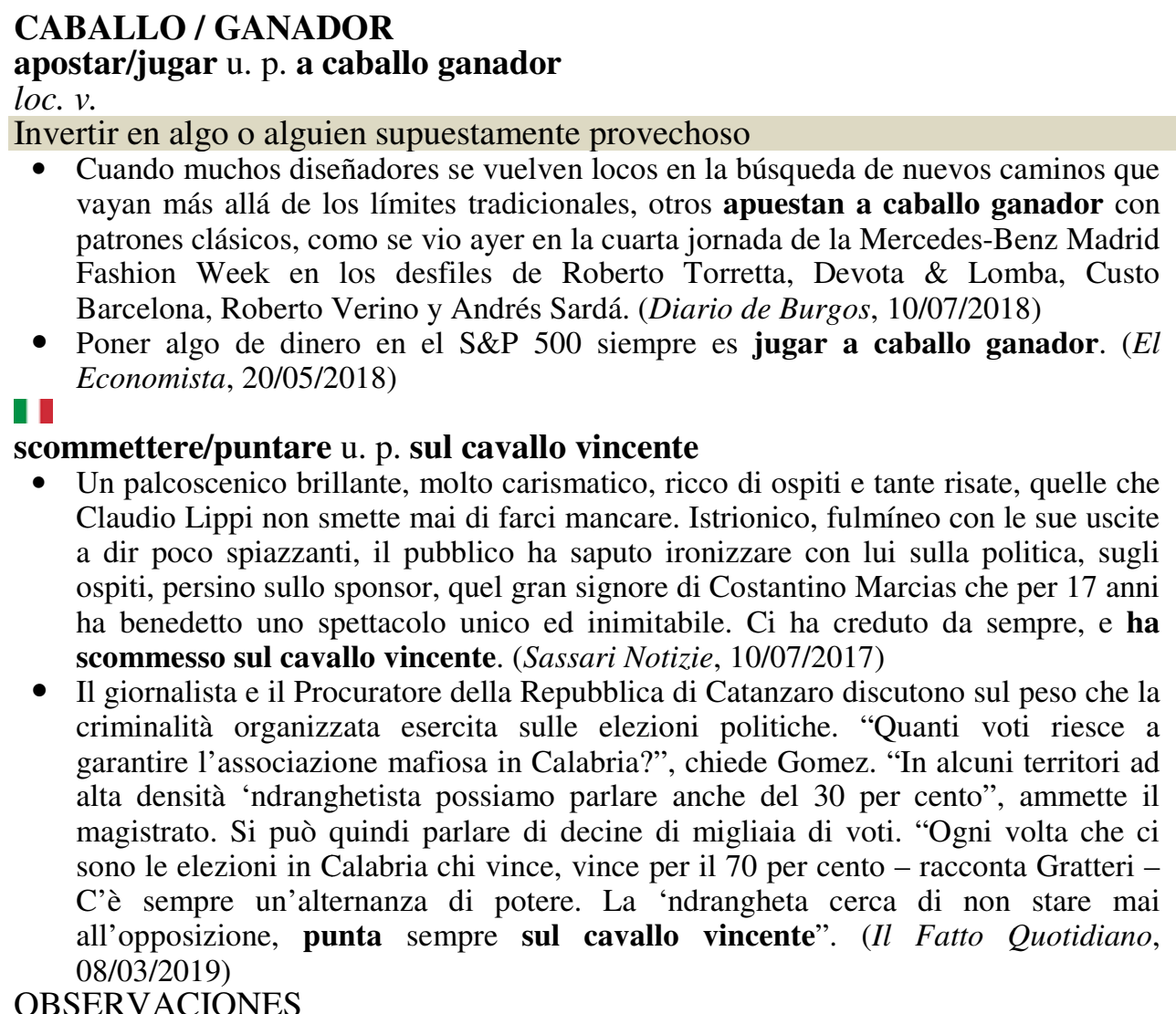

I Antónimos: scommettere/puntare u. p. sul cavallo perdente

$\mathrm{Si}$, por el contrario, el usuario buscase ese mismo fraseologismo bajo la entrada relativa a apostar, la posibilidad de conmutar dicho verbo con otro se señalaría situando jugar entre corchetes: 
APOSTAR

apostar [o jugar] u. p. a caballo ganador

loc. $v$.

Invertir en algo o alguien supuestamente provechoso

- Cuando muchos diseñadores se vuelven locos en la búsqueda de nuevos caminos que vayan más allá de los límites tradicionales, otros apuestan a caballo ganador con patrones clásicos, como se vio ayer en la cuarta jornada de la Mercedes-Benz Madrid Fashion Week en los desfiles de Roberto Torretta, Devota \& Lomba, Custo Barcelona, Roberto Verino y Andrés Sardá. (Diario de Burgos, 10/07/2018)

- Poner algo de dinero en el S\&P 500 siempre es jugar a caballo ganador. (El

1 Economista, 20/05/2018)

scommettere/puntare $u$. p. sul cavallo vincente

- Un palcoscenico brillante, molto carismatico, ricco di ospiti e tante risate, quelle che Claudio Lippi non smette mai di farci mancare. Istrionico, fulmíneo con le sue uscite a dir poco spiazzanti, il pubblico ha saputo ironizzare con lui sulla politica, sugli ospiti, persino sullo sponsor, quel gran signore di Costantino Marcias che per 17 anni ha benedetto uno spettacolo unico ed inimitabile. Ci ha creduto da sempre, e ha scommesso sul cavallo vincente. (Sassari Notizie, 10/07/2017)

- Il giornalista e il Procuratore della Repubblica di Catanzaro discutono sul peso che la criminalità organizzata esercita sulle elezioni politiche. "Quanti voti riesce a garantire l'associazione mafiosa in Calabria?", chiede Gomez. "In alcuni territori ad alta densità "ndranghetista possiamo parlare anche del 30 per cento", ammette il magistrato. Si può quindi parlare di decine di migliaia di voti. "Ogni volta che ci sono le elezioni in Calabria chi vince, vince per il 70 per cento - racconta Gratteri C'è sempre un'alternanza di potere. La 'ndrangheta cerca di non stare mai all'opposizione, punta sempre sul cavallo vincente". (Il Fatto Quotidiano, 08/03/2019)

OBSERVACIONES

II Antónimo: scommettere/puntare u. p. sul cavallo perdente

De manera parecida, la ficha que aparecerá bajo el verbo jugar es la siguiente:

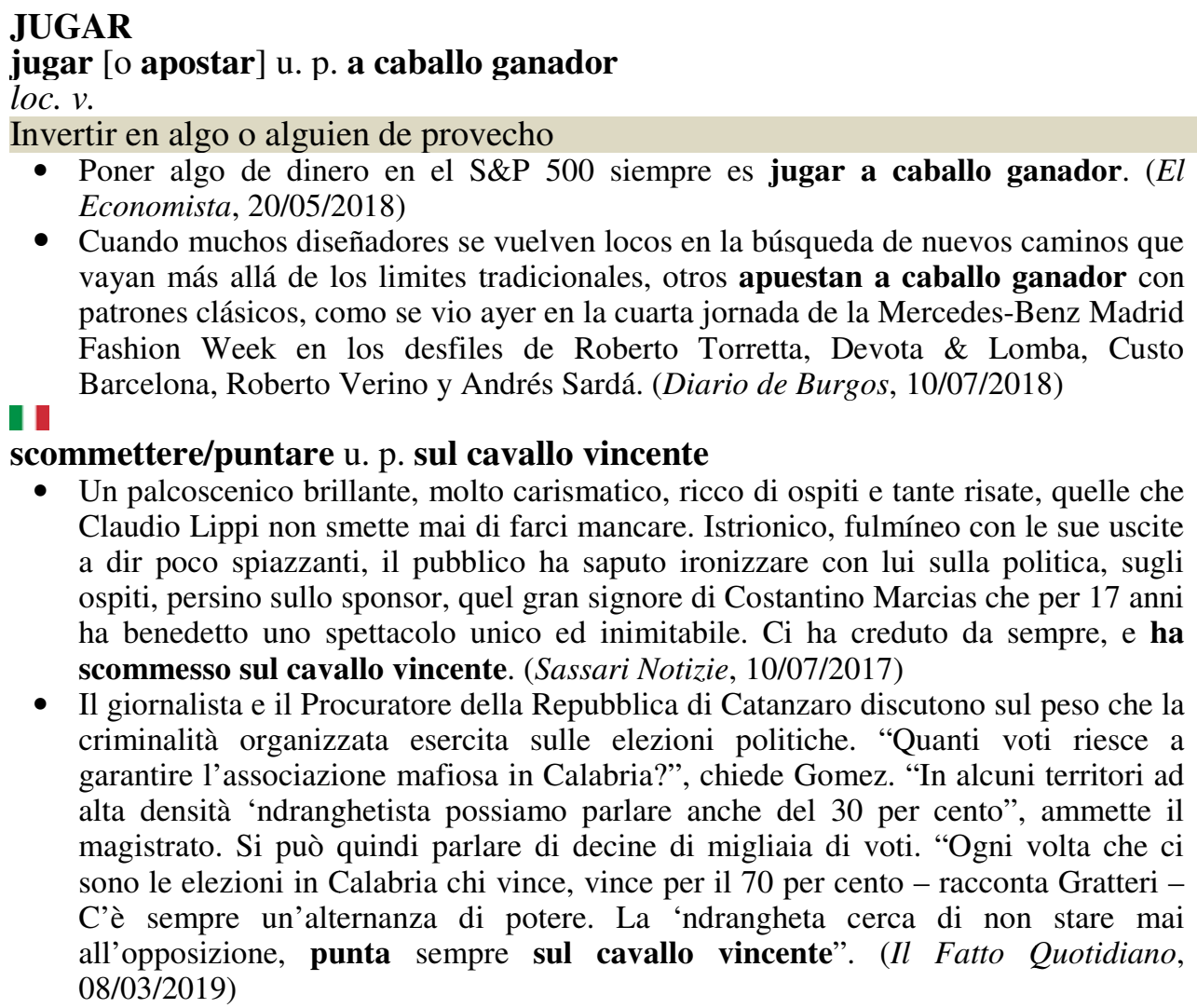

OBSERVACIONES

1 Antónimo: scommettere/puntare u. p. sul cavallo perdente

Como puede observarse, la única variación con respecto a la ficha anterior se halla en el orden de presentación de los dos fragmentos textuales españoles; debido a que la palabra clave de la consulta 
lexicográfica es, en este último caso, jugar, se propone primero la muestra de uso con dicho verbo y sucesivamente la que contiene la variante excluyente apostar. La misma estrategia se aplica a las fichas en que estos fenómenos atañen al italiano, como se aprecia en el ejemplo de it. scommettere/puntare sul cavallo vincente:

\section{SCOMMETTERE}

scommettere [o puntare] u. p. sul cavallo vincente

loc. $v$.

Investire su qualcuno o qualcosa in grado di portare benefici

- Un palcoscenico brillante, molto carismatico, ricco di ospiti e tante risate, quelle che Claudio Lippi non smette mai di farci mancare. Istrionico, fulmíneo con le sue uscite a dir poco spiazzanti, il pubblico ha saputo ironizzare con lui sulla politica, sugli ospiti, persino sullo sponsor, quel gran signore di Costantino Marcias che per 17 anni ha benedetto uno spettacolo unico ed inimitabile. Ci ha creduto da sempre, e ha scommesso sul cavallo vincente. (Sassari Notizie, 10/07/2017)

- Il giornalista e il Procuratore della Repubblica di Catanzaro discutono sul peso che la criminalità organizzata esercita sulle elezioni politiche. "Quanti voti riesce a garantire l'associazione mafiosa in Calabria?", chiede Gomez. "In alcuni territori ad alta densità "ndranghetista possiamo parlare anche del 30 per cento", ammette il magistrato. Si può quindi parlare di decine di migliaia di voti. "Ogni volta che ci sono le elezioni in Calabria chi vince, vince per il 70 per cento - racconta Gratteri C'è sempre un'alternanza di potere. La 'ndrangheta cerca di non stare mai all'opposizione, punta sempre sul cavallo vincente". (Il Fatto Quotidiano, 08/03/2019)

s

apostar/jugar u. p. a caballo ganador

- Cuando muchos diseñadores se vuelven locos en la búsqueda de nuevos caminos que vayan más allá de los límites tradicionales, otros apuestan a caballo ganador con patrones clásicos, como se vio ayer en la cuarta jornada de la Mercedes-Benz Madrid Fashion Week en los desfiles de Roberto Torretta, Devota \& Lomba, Custo Barcelona, Roberto Verino y Andrés Sardá. (Diario de Burgos, 10/07/2018)

- Poner algo de dinero en el S\&P 500 siempre es jugar a caballo ganador. (El Economista, 20/05/2018)

OSSERVAZIONI

\section{- Antonimo: apostar/jugar u. p. a caballo perdedor}

Las variantes facultativas, por su parte, se ubican entre paréntesis, siendo miembros opcionales; véase, a modo de ejemplo, la ficha relativa a it. (bella/brutta) gatta da pelare:

\section{GATTO, -A}

(bella/brutta) gatta da pelare

loc. n. [col.]

/persone, cose/

Problema o questione di difficile risoluzione

- Ed è anche sul futuro di queste due strutture che si gioca la partita: più di una volta il Comune ha sottolineato la necessità di avere un progetto che preveda una riconversione dei due ospedali attuali, per evitare che diventino degli scheletri abbandonati. Il precedente, anche in questo caso, è quello della Città della salute: il piano per realizzare il mega-ospedale non ha infatti previsto un riutilizzo dell'Istituto Tumori e del Besta. Con conseguente gatta da pelare (ancora oggi) sia per il Comune sia per la Regione. (La Repubblica, 21/08/2019)

- Gli antifurti satellitari sono una bella gatta da pelare per i ladri d'auto e così una startup nordica ha ben pensato di crearne una versione per bici. (Il Sole 24 ORE, 03/09/2018)

- "Sarà una gara difficile, l'Udinese è un'ottima squadra, fisica, che difende bene e ha giocatori di gamba molto forti nelle ripartenze. Dovremo fare attenzione a Lasagna e alla qualità di altri giocatori come Pussetto, Nestorovski, De Paul. Grande attenzione anche sui calci da fermo. L'Udinese è una brutta gatta da pelare, ma ci stiamo preparando nel modo giusto". (Corriere Adriatico, 13/09/2019)

\section{hueso duro/difícil de roer}

/persone, cose/

- El entrenador del Rayo Majadahonda, Antonio Iriondo, consideró en vísperas de la visita al Ramón de Carranza que "el Cádiz es un hueso duro de roer", aunque 
destacó que su equipo mejora a cada jornada que pasa y que poco a poco se va cohesionando tras realizar numerosos fichajes el pasado verano. (La Vanguardia, $08 / 12 / 2018$ )

- Los 52 chalés de lujo ilegales de O Grove que la Xunta quiere salvar [...] En plena burbuja inmobiliaria, la Xunta del bipartito apercibió al constructor e inició los trámites que acabaron en una inevitable orden de derribo con el Gobierno del PP, que se encontró con un hueso difícil de roer. (El País, 01/01/2019)

\section{OSSERVAZIONI}

\section{¿ Antonimi: juego de niños, pan comido}

Los fragmentos textuales presentes en la ficha ejemplifican el uso tanto de gatta da pelare como de bella gatta da pelare y brutta gatta da pelare. Los dos antónimos bella y brutta pierden en esta UF su significado calificativo; de ahí la plena coincidencia funcional de las tres variantes lematizadas (gatta da pelare, bella gatta da pelare, brutta gatta da pelare).

\subsection{Sinonimia}

Al tratar de establecer correspondencias interlingüísticas, es posible observar que la sinonimia puede llegar a manifestarse de manera unilateral o bilateral: en el primer caso, denominado homonimia fraseológica por Navarro (2008: 89), a una locución de una lengua corresponderían más equivalentes funcionales, todos sinónimos, en la otra lengua. En cambio, una relación de sinonimia bilateral supondría la presencia de dos o más expresiones sinónimas en ambos idiomas. Un ejemplo del primer tipo lo facilitan el fraseologismo it. partire per la tangente y sus tres equivalentes esp. escaparse/irse/salirse por la tangente, irse por las ramas e irse por los cerros de Úbeda.

Veamos ahora la lematización de it. partire per la tangente:

\section{TANGENTE}

\section{partire $u$. p. per la tangente}

loc. v. [col.]

Trattare temi secondari per evitare di affrontare una questione importante

- Invece di partire per la tangente - attacca Aramini - con accuse, da parte della consigliera Zuliani (ma parla a nome personale o del Pd?), sarebbe stato meglio entrare nel merito delle finalità della delibera. (Latina Corriere, 23/04/2019)

- Poi però, quando gli viene chiesto se la squadra stavolta ha avuto la "cazzimma" che era mancata a Madrid, De Laurentiis parte per la tangente: "Mi fa piacere che il termine da me usato l'altra volta sia stato usato dalla Gazzetta dello Sport presentando la partita, evidentemente la ricchezza dei nostri dialetti è unica e può assurgere a dignità di lingua nazionale, cazzimma è una parola bella, riempie la bocca di gioia”. (La Repubblica, 08/03/2017)

escapar(se)/ir(se)/salir(se) u. p. por la tangente

- El cantante portugués escapa por la tangente de cualquier tópico eurovisivo en $\mathrm{La}$ Mar de Músicas de Cartagena. (El Diario, 27/07/2018)

- Rajoy se va por la tangente: ni una palabra de Gürtel pero sí de terrorismo. El presidente del Gobierno presume en el Congreso de lucha anticorrupción y centra el debate en sus "prioridades": los atentados de Barcelona y Cambrils, el independentismo en Catalunya y la economía. (Público, 30/08/2017)

- Al respecto, Ruiz ha considerado "irrisorio" que el presidente del PP onubense haya declarado este lunes que "todo se deba a una estrategia del PSOE", porque eso es "salirse por la tangente y no saber afrontar ni querer un problema serio interno que tiene el PP de Huelva, tal es no poder controlar lo que sus dirigentes van diciendo por ahí, según les convenga en cada momento y con cada cuestión concreta". ( $L a$ Vanguardia, 15/07/2019)

\section{andarse/irse $u$. p. por las ramas}

- Ignacio Mariscal es el CEO de Reale, así que no nos andamos por las ramas y le preguntamos por qué su compañía es un buen lugar para trabajar. (El Mundo, 26/09/2019)

- - ¿Para qué? Seguro que pagan todos. Le hice una insinuación al mayor de los Arrizabalaga. Ya vi que se iba por las ramas. Estos asuntos, como te he dicho, los soluciona cada cual por su cuenta. (CORPES XXI, Aramburu, Patria, 2016)

irse u. p. por los cerros de Úbeda

- En la sala de prensa (en la que los ganadores de los premios responden a las preguntas de periodistas pocos minutos después de recoger su galardón) sí que salió esta cuestión. Era inevitable. Al ser preguntado por Singer, el productor Graham 
King respondió: "Eso no es algo de lo que deberíamos hablar esta noche". Rami Malek, ante la misma pregunta, se fue por los cerros de Úbeda: "Solo había una cosa que necesitábamos hacer: celebrar a Freddie Mercury". (El País, 14/01/2019)

- En vistas de que todo el mundo habla del gobierno (del que hay y del que está en ser), habíamos pensado desmarcarnos e irnos por los cerros de Úbeda para hablar de cualquier fútil chorrada. (El Diario de Almería, 01/07/2019)

OSSERVAZIONI

I Antonimo: ir u. p. al grano

En la ficha propuesta aparecen las tres UF sinónimas del español, todas en negrita y seguidas por dos muestras de uso. De esta manera, el usuario tiene la oportunidad de comprobar la efectiva equivalencia funcional de los sinónimos propuestos y de elegir entre ellos el que considere más oportuno para sus fines comunicativos. Como puede observarse, si la lengua meta cuenta con un equivalente funcional muy parecido desde el punto de vista estructural, léxico y/o icónico al fraseologismo de la lengua de búsqueda, se ofrecerá primero este y sucesivamente los demás sinónimos.

En caso de que se llevase a cabo una búsqueda en la dirección opuesta (español > italiano), la ficha incluiría una de las tres locuciones españolas —andarse/irse por las ramas, en el ejemplo que se detalla a continuación - y el equivalente italiano partire per la tangente:

\section{RAMA}

\section{andarse/irse $u$. p. por las ramas}

loc. v. [col.]

Hablar de un tema menor para no tener que hablar de un asunto más importante

- En esta ocasión, la interrupción de Manuel Marchena solo pretende ayudar a Jordi Pina a centrar a un testigo con tendencia a irse por las ramas, pero también es cierto -como suelen decir los testigos cuando intentan añadir algo que no se les ha preguntado- que durante los últimos días se ha producido un incremento de los choques entre los abogados defensores y el presidente del tribunal. (El País, 21/03/2019)

- —Para qué? Seguro que pagan todos. Le hice una insinuación al mayor de los Arrizabalaga. Ya vi que se iba por las ramas. Estos asuntos, como te he dicho, los soluciona cada cual por su cuenta. (CORPES XXI, Aramburu, Patria, 2016)

11

partire u. p. per la tangente

- Invece di partire per la tangente - attacca Aramini - con accuse, da parte della consigliera Zuliani (ma parla a nome personale o del Pd?), sarebbe stato meglio entrare nel merito delle finalità della delibera. (Latina Corriere, 23/04/2019)

- Poi però, quando gli viene chiesto se la squadra stavolta ha avuto la "cazzimma" che era mancata a Madrid, De Laurentiis parte per la tangente: "Mi fa piacere che il termine da me usato l'altra volta sia stato usato dalla Gazzetta dello Sport presentando la partita, evidentemente la ricchezza dei nostri dialetti è unica e può assurgere a dignità di lingua nazionale, cazzimma è una parola bella, riempie la bocca di gioia”. (La Repubblica, 08/03/2017)

OBSERVACIONES

Antónimos: arrivare u. p. al dunque, venire u. p. al sodo

Por tanto, en este caso la equivalencia interlingüística queda reducida a una relación aparentemente biunívoca, al estimarse innecesaria la inclusión de los sinónimos españoles escapar(se)/ir(se)/salir(se) por la tangente e irse por los cerros de Úbeda.

De manera parecida, si el usuario buscase un equivalente italiano de irse por los cerros de Úbeda se hallaría ante la ficha siguiente, en la que no se mencionan ni escapar(se)/ir(se)/salir(se) por la tangente ni andarse/irse por las ramas, puesto que dicha serie de UF sinónimas en español se facilita en la ficha que resulta de una búsqueda en la dirección contraria (es decir, del italiano al español):

\section{CERRO / IR}

irse u. p. por los cerros de Úbeda

loc. v. [col.]

Hablar de un tema menor para no tener que hablar de un asunto más importante

- En la sala de prensa (en la que los ganadores de los premios responden a las preguntas de periodistas pocos minutos después de recoger su galardón) sí que salió esta cuestión. Era inevitable. Al ser preguntado por Singer, el productor Graham King respondió: "Eso no es algo de lo que deberíamos hablar esta noche". Rami Malek, ante la misma pregunta, se fue por los cerros de Úbeda: "Solo había una 
cosa que necesitábamos hacer: celebrar a Freddie Mercury". (El País, 14/01/2019)

- En vistas de que todo el mundo habla del gobierno (del que hay y del que está en ser), habíamos pensado desmarcarnos e irnos por los cerros de Úbeda para hablar de cualquier fútil chorrada. (El Diario de Almería, 01/07/2019)

1

partire $u$. p. per la tangente

- Invece di partire per la tangente - attacca Aramini - con accuse, da parte della consigliera Zuliani (ma parla a nome personale o del Pd?), sarebbe stato meglio entrare nel merito delle finalità della delibera. (Latina Corriere, 23/04/2019)

- Poi però, quando gli viene chiesto se la squadra stavolta ha avuto la "cazzimma" che era mancata a Madrid, De Laurentiis parte per la tangente: "Mi fa piacere che il termine da me usato l'altra volta sia stato usato dalla Gazzetta dello Sport presentando la partita, evidentemente la ricchezza dei nostri dialetti è unica e può assurgere a dignità di lingua nazionale, cazzimma è una parola bella, riempie la bocca di gioia”. (La Repubblica, 08/03/2017)

OBSERVACIONES

Antónimos: arrivare u. p. al dunque, venire u. p. al sodo

Como ya hemos adelantado, la sinonimia puede llegar a manifestarse simultáneamente en ambos idiomas; véase, por ejemplo, el conjunto de las UF que suelen ser más habituales para expresar el significado 'marcharse muy deprisa', que cuenta con poner pies en polvorosa, salir por patas/piernas/pies y coger/tomar las de Villadiego en español y con darsela a gambe, fuggire/scappare a gambe levate y tagliare la corda en italiano. En casos como este, todas las búsquedas llevadas a cabo, tanto del español al italiano como del italiano al español, darían como resultado una ficha en la que aparecerían dos o más equivalentes funcionales. Repárese, verbigracia, en la entrada de esp. salir por patas/piernas/pies:

\section{PATA}

salir u. p. por patas [o piernas o pies]

loc. v. [col.]

Marcharse muy deprisa

- Puigdemont sale "por patas" y destroza a Podemos: "No tenían ni media bofetada". (Libertad Digital, 31/10/2017)

- La paradoja ha querido que cierre el círculo el hombre que se negó a abrirlo, el mismo Marcelino que días después de aquella victoria sin brindis de 2008 vino, vio y salió por piernas al descubrir que el proyecto ganador que le habían vendido se erigía sobre una gran mentira. (Las Provincias, 23/05/2019)

- O, incluso de forma más directa: ¿había llegado la hora de salir por pies de Estados Unidos y dejárselo a... quién exactamente? No lo sabemos. ¿Para ir a dónde exactamente? ¿A Europa? Genial. (El País, 26/11/2018)

11

darsela u. p. a gambe

- La classe dirigente? Se l'è data a gambe. Industriali, vescovi, magistrati, sindacalisti, intellettuali: un tempo la politica temeva le élite. Ma con l'avvento dei populisti, accade il contrario. (La Stampa, 11/12/ 2018)

- Darsela a gambe. Non erano quaranta, come i ladroni. Sarebbero addirittura cinquanta i migranti eritrei sbarcati dalla Diciotti e scomparsi dalla struttura di Rocca Di Papa. (Il Foglio, 05/09/2018)

fuggire/scappare $u$. p. a gambe levate

- Parliamo dell'esordio di Zhang Chi, che firma anche la sceneggiatura del suo The Shaft, film in tre episodi che raccontano le vicende di un padre e dei suoi due figli, una ragazza e un ragazzo, vicende che non si intrecciano se non in maniera molto marginale. Il tutto tenendo sullo sfondo, ma in maniera vivida e sempre visibile e palpabile, l'elemento che muove i fili di tutto il film, ovvero la miniera dalla quale tutti i nostri protagonisti vogliono fuggire a gambe levate. (PAISÀ, Cineblog, consulta: 31/10/2019)

- La fidanzata acciuffa il bouquet, lui scappa dal matrimonio a gambe levate. ( $\mathrm{La}$ Gazzetta dello Sport, 30/09/2019)

tagliare u. p. la corda

- Tunisini "fantasma" cercano di tagliare la corda: bloccati a Porto Empedocle. (Agrigento Notizie, 03/05/2019)

- Hanno rubato tre trattori e hanno tagliato la corda senza fare il minimo rumore. Il maxi-furto è stato messo a segno nella notte tra giovedì e venerdì ai danni dell'azienda agricola Borga Virginio e Figli di via Roma. (Il Gazzettino, 16/12/2018)

OBSERVACIONES

a Sinónimos: poner u. p. pies en polvorosa, tomar/coger u. p. las de Villadiego 
En el apartado final de observaciones se introducen los sinónimos españoles de salir por patas/piernas/pies al fin de ofrecer un cuadro lo más exhaustivo posible de las relaciones de sinonimia bilateral que se dan en este caso.

Símilmente, al buscar un equivalente funcional de it. darsela a gambe el usuario se hallaría ante la ficha siguiente:

GAMBA

darsela u. p. a gambe

loc. v. [col.]

Andarsene di corsa, rapidamente

- La classe dirigente? Se l'è data a gambe. Industriali, vescovi, magistrati, sindacalisti, intellettuali: un tempo la politica temeva le élite. Ma con l'avvento dei populisti, accade il contrario. (La Stampa, 11/12/ 2018)

- Darsela a gambe. Non erano quaranta, come i ladroni. Sarebbero addirittura cinquanta i migranti eritrei sbarcati dalla Diciotti e scomparsi dalla struttura di Rocca Di Papa. (Il Foglio, 05/09/2018)

salir u. p. por patas/piernas/pies

- Puigdemont sale "por patas" y destroza a Podemos: "No tenían ni media bofetada". (Libertad Digital, 31/10/2017)

- La paradoja ha querido que cierre el círculo el hombre que se negó a abrirlo, el mismo Marcelino que días después de aquella victoria sin brindis de 2008 vino, vio y salió por piernas al descubrir que el proyecto ganador que le habían vendido se erigía sobre una gran mentira. (Las Provincias, 23/05/2019)

- O, incluso de forma más directa: ¿había llegado la hora de salir por pies de Estados Unidos y dejárselo a... quién exactamente? No lo sabemos. ¿Para ir a dónde exactamente? ¿A Europa? Genial. (El País, 26/11/2018)

poner $u$. p. pies en polvorosa

- Javier Beirán también pone pies en polvorosa. El alero madrileño se acoge a la cláusula de salida para finalizar su vínculo con el Iberostar Tenerife y firmar en Gran Canaria dos temporadas. (El Diario de Avisos, 29/06/2019)

- BBVA pone pies en polvorosa y se lleva 9.600 millones en bonos de Londres a Dublín. (Valencia Plaza, 12/04/2019)

tomar/coger $u$. p. las de Villadiego

- Todos aquellos que tomaron las de Villadiego obligados por las circunstancias triunfaron fuera de casa como ocurrió con Urruticoechea o Ansola, ambos realistas, pero si hubo relevos que causaron impacto como en el Madrid el cambio de Juanito Alonso por Domínguez o el complicado caso en que convivieron en el Bernabéu, Alonso, Domínguez, Vicente y Bagur. (República, 03/10/2019)

- Descubierta la cosa, y hecho un ridículo monumental, y con varios informes médicos que avalaban que Zaida no estaba buena, que no estaba bien, que no estaba en forma, vaos, que no funcionaba, Zaida optó por salir pitando, coger las de Villadiego y retirarse; y es que se le acabó el amor al uniforme de tanto usarlo. (El Español Digital, 12/03/2018)

OSSERVAZIONI

I Sinonimi: fuggire/scappare u. p. a gambe levate, tagliare u. p. la corda

\section{Conclusiones}

La propuesta fraseográfica que se ha perfilado en este ensayo confirma, según creemos, la incidencia positiva de las herramientas digitales no solamente en los procesos de elaboración de diccionarios sino también en la fruición de estos por parte de los usuarios. De hecho, gracias al formato electrónico es posible dar cuenta de las variantes léxicas sea en la forma canónica de los fraseologismos sea en los fragmentos textuales que sirven de ejemplos en la microestructura de las entradas; así pues, cuantas más sean las variantes, mayor será el número de muestras presentes en la ficha.

Por otra parte, la aplicación de las ciencias informáticas a la práctica lexicográfica permite proponer una representación abarcadora de las relaciones de sinonimia $-\mathrm{y}$, cabría añadir, aunque en menor medida, de antonimia - que se establecen entre las UF de los dos idiomas considerados, lo cual representa una ventaja de relieve en el caso de un diccionario bilingüe o multilingüe dirigido sobre todo a estudiantes y traductores.

Quedan, por supuesto, muchas cuestiones por resolver; entre ellas, la lematización de "casos fronterizos" (García-Page Sánchez 2008: 221) como el binomio it. andare/venire al sodo y arrivare/venire al dunque, cuya clasificación y consiguiente inclusión en nuestro diccionario podría definirse con razón un hueso 
difícil/duro de roer, debido a las dificultades que suscita su ubicación dentro del fenómeno de la variación o bien de la sinonimia. Otro reto lo constituyen pares como esp. apear [alguien] del burro [a alguien] y apearse [alguien] del burro, puesto que la valencia sintáctica de las dos UF repercute en sus significados (respectivamente ‘cambiar de opinión' y ‘conseguir que alguien cambie de opinión'), planteando así dudas sobre su tratamiento lexicográfico: ¿se trata de variantes, de sinónimos o bien de dos fraseologismos distintos?

Estos desafíos, que paso a paso se están solucionando, ponen de relieve una vez más la incidencia que una metodología como la que hemos introducido aquí tendría en el panorama lexicográfico actual, al contribuir a acortar las distancias entre las reflexiones teóricas delineadas en el marco contrastivo entre español e italiano y la producción fraseográfica centrada en las correspondencias entre estos dos idiomas, muy escasa hasta la fecha y monodireccional (del español al italiano). Asimismo, el repertorio lexicográfico planteado ampliaría, enriqueciéndolo, el exiguo abanico de obras en línea y de libre acceso - p. ej. el Dictionnaire d'expressions idiomatiques (Français-Portugais-Français) de Claudia Maria Xatara y el Deutsch-türkische Wortverbindungen Wirtschaft de Ayfer Aktaş - de los que disponen actualmente los cibernautas interesados en cuestiones relativas al ámbito de la traducción o del aprendizaje de lenguas extranjeras.

\section{Agradecimientos}

El propósito de confeccionar un diccionario fraseológico en línea se ha concebido en el marco del proyecto de investigación El discurso repetido: análisis cognitivo, contrastivo, traductivo e histórico-cultural de las unidades fraseológicas de la Universidad de Verona y, sucesivamente, se ha afianzado en la Universidad Ca' Foscari de Venecia, tras el reconocimiento ministerial del Dipartimento di Studi Linguistici e Culturali Comparati como "Dipartimento di Eccellenza 2018-2022" en el ámbito de una convocatoria del Ministerio italiano de Educación, Universidad e Investigación.

\section{Bibliografía}

\section{Estudios}

Águila Escobar, Gonzalo (2009): Los diccionarios electrónicos del español. Madrid: Arco/Libros.

Álvarez de la Granja, María (1999): "Variación e sinonimia nas unidades fraseolóxicas. Caracterización xeral e proposta de tratamento lexicográfico". Cadernos de Lingua, n. 19, pp. 43-64.

Autelli, Erica; Konecny, Christine; Lusito, Stefano (2019): "GEPHRAS: il primo dizionario combinatorio genoveseitaliano online”. En Fiorenzo Toso (ed.), Il patrimonio linguistico storico della Liguria: attualità e futuro. Raccolta di Studi, Savona: InSedicesimo, pp. 21-32.

Capra, Daniela (2016a): "La marcación de la fraseología en diccionarios italiano-español y español-italiano". Paremia, n. 25 , pp. 45-59.

Capra, Daniela (2016b): "Il metalinguaggio della fraseologia in dizionari e testi specialistici tra Italia e Spagna". En Elena Dal Maso, Carmen Navarro (eds.), Gutta cavat lapidem. Indagini fraseologiche e paremiologiche, Mantova: Universitas Studiorum, pp. 269-284.

Corpas Pastor, Gloria (1996): Manual de fraseología española. Madrid: Gredos.

Dal Maso, Elena (2019): "Estudio funcional y lexicográfico de la polisemia simétrica y asimétrica en un corpus bilingüe de locuciones meteorológicas del español y del italiano". Lingue e Linguaggi, n. 33, pp. 41-53.

Fajardo Rojas, Carmen Teresa (2017): "Sinonimia en locuciones léxicas del español mexicano". Anuario de Letras. Lingüística y Filología, vol. V, n. 2, pp. 91-112.

García-Page Sánchez, Mario (2008): Introducción a la fraseología española. Estudio de las locuciones. Barcelona: Anthropos.

García-Page Sánchez, Mario (2016): "Variantes léxicas correlativas". En Elena Dal Maso, Carmen Navarro (eds.), Gutta cavat lapidem. Indagini fraseologiche e paremiologiche, Mantova: Universitas Studiorum, pp. 39-54.

Gelpí Arroyo, Cristina (2003): "El estado actual de la lexicografía: los nuevos diccionarios”. En Antonia María Medina Guerra (coord.), Lexicografía española, Barcelona: Ariel Lingüística, pp. 353-385.

Imperiale, Riccardo; Schafroth, Elmar (2019): "Fraseologia italiana basata sull'uso: lessicografia digitale per apprendenti tra la frame semantics e la grammatica delle costruzioni”. Italiano LinguaDue, vol. XI, n. 1, pp. 1-28.

Larreta Zulategui, Juan Pablo (2001): Fraseología contrastiva del alemán y del español. Frankfurt am Main: Peter Lang. 
Lončar, Ivana; Dal Maso, Elena (2018): "Propuesta de elaboración lexicográfica de la equivalencia y polisemia asimétricas en un diccionario fraseológico multilingüe en línea: el caso de español, italiano y croata”. En Antonio Pamies, Alexandra Magdalena, Isabel M. ${ }^{a}$ Balsas (eds.), Lenguaje figurado y competencia interlingüística (II). Aplicaciones lexicográficas y traductológicas, Granada: Editorial Comares (Interlingua, n. 201), pp. 29-51.

Marello, Carla (2017): Lexicografía digital y enseñanza de lenguas / Lessicografia digitale e insegnamento delle lingue, Madrid, Universidad Complutense de Madrid, Tesis Doctoral dirigida por Margarita Natalía Borreguero Zuloaga.

Mellado Blanco, Carmen (2015): "Parámetros específicos de equivalencia en las unidades fraseológicas (con ejemplos del español y el alemán)”. Revista de filología, n. 33, pp. 153-174.

Montoro del Arco, Esteban (2004): "La variación fraseológica y el diccionario", en Paz Battaner, Janet DeCesaris (eds.), De Lexicografia (Actes del I Symposium Internacional de Lexicografia), Barcelona: Institut Universitari de Lingüística Aplicada, pp. 591-604.

Navarro, Carmen (2008): Aspectos de fraseología contrastiva. Verona: Fiorini.

Nomdedeu-Rull, Antoni; Tarp, Sven (2018): "Hacia un modelo de diccionario en línea para aprendices de español como LE/L2”. Journal of Spanish Language Teaching, vol. V, n. 1, pp. 50-65. DOI: 10.1080/23247797.2018.1459279.

Penadés Martínez, Inmaculada (2007): “Sinonimia y locuciones”. En Pablo Cano López (ed.), Actas del VI Congreso de Lingüística General, vol. II, t. 2, Madrid: Arco/Libros, pp. 2217-2228.

Quiroga Munguía, Paula (2005): "Fraseología y lexicografía: el tratamiento de la fraseología en los diccionarios bilingües generales italiano/español del siglo XX”. Revista de lexicografía, n. 11, pp. 105-147.

Rodríguez-Piñero Alcalá, Ana Isabel (2011): "La sinonimia y la antonimia en el marco de un diccionario de locuciones". Lingüística en la Red, n. 9, pp. 1-27.

Rodríguez-Piñero Alcalá, Ana Isabel (2012): "Variación y sinonimia en las locuciones". Revista de Lingüística y Lenguas Aplicadas, n. 7, pp. 225-236.

San Vicente, Félix (dir.): Portal de lingüística contrastiva español-italiano. En línea: http://www.contrastiva.it/wp/ [consulta: 20/01/2020].

Valero Gisbert, María (2010): "Determinación del significado de UUFF en los DDBB italiano-español actuales". En Marta C. Ayala Castro, Antonia María Medina Guerra (coords.), Diversidad lingüística y diccionario, Málaga: Universidad de Málaga (Estudios y Ensayos, n. 123), pp. 417-437.

Valero Gisbert, María (2012): Fraseología, gramática, lexicografía. Mantova: Universitas Studiorum (col. Strumenti).

Valero Gisbert, María (2015): "Tratamiento lexicográfico de unidades fraseológicas desde la perspectiva de la marcación pragmática”. Cuadernos AISPI: Estudios de lenguas y literaturas hispánicas, n. 6, pp. 37-52.

Valero Gisbert, María (2017): "Fraseología y marcas diacrónicas en la lexicografía bilingüe de los diccionarios más representativos de español/italiano del s. XXI”. En Cecilio Garriga Escribano, Ignacio Sariego López, Juan Gutiérrez Cuadrado (eds.), El diccionario en la encrucijada: de la sintaxis y la cultura al desafío digital, Santander: Escuela Universitaria de Turismo Altamira, pp. 395-412.

Valero Gisbert, María (2019): "En torno al ejemplo fraseológico en la lexicografía bilingüe italoespañola". Revista de filología española, vol. XCIX, n. 1, pp. 211-225.

Zuluaga Ospina, Alberto (1980): Introducción al estudio de las expresiones fijas. Frankfurt am Main: Peter Lang.

\section{Diccionarios y corpus textuales}

Aktas, Ayfer: Deutsch-türkische Wortverbindungen Wirtschaft. En línea: http://wvonline.ids-mannheim.de/ dtww/index.htm [consulta: 01/02/2020].

Arqués, Rossend; Padoan, Adriana (2012): Il grande dizionario di spagnolo. Dizionario spagnolo-italiano, italianospagnolo. Bologna: Zanichelli.

Buitrago, Alberto (2009): Diccionario de dichos y frases hechas, 3. a ed. Madrid: Espasa Calpe.

Casares, Julio (1942): Diccionario ideológico de la lengua española. Madrid: Gredos.

CORPES XXI = Real Academia Española: Corpus del Español del Siglo XXI. En línea: http://www.rae.es/recursos/ banco-de-datos/corpes-Xxi [consulta 09/10/ 2019].

DiLEA = Penadés Martínez, Inmaculada (2019): Diccionario de locuciones idiomáticas del español actual. En línea: http://www.diccionariodilea.es/ [consulta 08/10/ 2019].

$D R A E$ = Real Academia Española (2001): Diccionario de la Lengua Española, 22. ${ }^{\text {ed. }}$ Madrid: Espasa Calpe.

$D L E$ = Real Academia Española (2014): Diccionario de la Lengua Española. En línea: https://dle.rae.es/ [consulta: 08/10/2019].

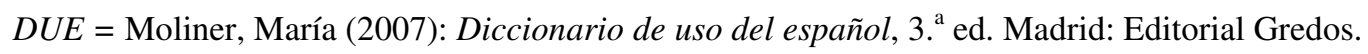

GRADIT = De Mauro, Tullio (dir.) (1999-2007): Grande dizionario italiano dell'uso. Milano: UTET.

Greco, Simone (2019): Diccionario combinatorio español italiano (A-K). Ogliastro Cilento: Licosia. 
PAISÀ = Scalise Sergio; Pirrelli, Vito (coords.) (2009-2012): Piattaforma per l'Apprendimento dell'Italiano Su corpora Annotati (PAISÀ). En línea: http://www.corpusitaliano.it/ [consulta 08/10/ 2019].

Pittano, Giuseppe (2009): Frase fatta capo ha. Dizionario dei modi di dire, proverbi e locuzioni. Bologna: Zanichelli.

Quartu, B. Monica; Rossi, Elena (2012): Dizionario dei modi di dire della lingua italiana. 10.000 modi di dire ed estensioni figurate in ordine alfabetico per lemmi portanti e campi di significato, 2. ${ }^{\mathrm{a}}$ ed. Milano: Rizzoli.

Sañé, Secundí; Schepisi, Giovanna (2013): Spagnolo idiomatico. Dizionario spagnolo italiano di frasi idiomatiche, colloquiali e gergali. Bologna: Zanichelli.

Seco, Manuel; Andrés, Olimpia; Ramos, Gabino (1999): Diccionario español actual. Madrid: Aguilar.

Seco, Manuel; Andrés, Olimpia; Ramos, Gabino (2004): Diccionario fraseológico documentado del español actual. Madrid: Aguilar.

Sorge, Paola (2011): Dizionario dei modi di dire della lingua italiana, 3. ${ }^{\text {a }}$ ed. Roma: Newton Compton.

Tam, Laura (2004): Dizionario spagnolo-italiano. Diccionario italiano-español, 3. ${ }^{\mathrm{a}}$ ed. Milano: Hoepli.

Varela, Fernando; Kubarth, Hugo (2004): Diccionario fraseológico del español moderno, 2. ${ }^{\text {a }}$ ed. Madrid: Gredos.

Vocabolario Treccani (2008). En línea: http://www.treccani.it/vocabolario/ [consulta 10/10/ 2019].

Xatara, Claudia Maria: Dictionnaire d'expressions idiomatiques (Français - Portugais - Français). En línea: https://www.cnrtl.fr/dictionnaires/expressions_idiomatiques/ [consulta: 01/02/2020]. 